\title{
Prevalence of dyslipidemia among climacteric women
}

\begin{abstract}
Background: The climacteric phase implies metabolic and hormonal changes that might lead to adverse effects, such as dyslipidemia and cardiovascular risks.
\end{abstract}

Objective: Determine factors associated with the prevalence of dyslipidemia among climacteric women treated in a reference clinic in southern Brazil.

Method: Cross-sectional study with 550 women aged 40-65 years treated in a clinic specialized in climacteric therapies and gynecologic surgeries. A precoded and pretested standardized survey with questions about socioeconomic, demographic, behavioral and reproductive features was applied. Laboratory tests needed to define lipid profiles were the analysis of HDL cholesterol, total cholesterol, and triglycerides. Multivariate analysis $(p<0.05)$ was used in the evaluation of data.

Results: 550 climacteric women were evaluated. Elevated prevalence of dyslipidemia $(83.8 \%)$ was found. The group presenting the largest number of dyslipidemic women was the group aged 56-65 years $(92.5 \%)$. There was no significant association between the occurrence of dyslipidemia and menopausal state. Multivariate analysis revealed that dyslipidemias were $11 \%$ more prevalent in women aged $56-65$ years (RP $1.11 ; \mathrm{CI}_{95 \%} 1.01$ to $1.21 \mathrm{p}=0.03$ ) and $16 \%$ more frequent among women with three or more gestational periods $(p=0.04)$. The prevalence of dyslipidemia was also higher among obese women than among the eutrophic ones, however, the significance level remained in a boundary zone (PR 1.12; $\mathrm{CI}_{95 \%} 1.00$ to $1.26 \mathrm{p}=0.06$ ).

Conclusion: A higher prevalence of dyslipidemia was found among women with multiple pregnancies, something that may be linked to hormonal and metabolic issues, as well as dietary issues during pregnancy. The menopausal state was not associated with the occurrence of dyslipidemia in this study. Possibly, the aging process and its metabolic changes - more than the menopausal state itself - is the main factor associated with dyslipidemia during the climacteric years.

Keywords: climacteric, menopause, dyslipidemia, women
Volume 6 Issue 6 - 2019

\author{
Juliana Granzotto de Vargas,' Renata \\ Miecinikovsski,' Heloísa Theodoro,' Dino \\ Roberto Soares De Lorenzi,' Omarwalid \\ Noorzada, ${ }^{2}$ Maria Teresa Anselmo Olinto, ${ }^{3}$ \\ Karina Giane Mendes' \\ 'Department of Nutrition, University of Caxias do Sul, Caxias \\ do Sul, Brazil \\ ${ }^{2}$ Public Health, Georgia State University, Georgia \\ ${ }^{3}$ Universidade do Vale do Rio dos Sinos (UNISINOS), \\ Universidade Federal do Rio Grande do Sul (UFRGS), Brazil
}

Correspondence: Heloísa Theodoro, Department of Nutrition, University of Caxias do Sul, Caxias do Sul, Brazil, Email htheodo@ucs.br

Received: October 18, 2019 | Published: November 13, 2019
Abbreviations: LDL, low-density lipoproteins; CVD, cardiovascular disease; BMI, body mass index.

\section{Introduction}

Climacteric is a transition phase in women's life, changing from a reproductive period into a non-reproductive one. It happens with endocrinal changes due to an ovarian follicular depletion, leading to a definitive hypoestrogenism with menstrual cycle disorders until its cessation (menopause). ${ }^{1}$ It begins around the age of 35 and 40 years, stretching out until 65 years. ${ }^{2}$ Among the climacteric hypoestrogenism consequences, literature has underlined its association with an increase in low-density lipoproteins (LDL) and high-density lipoproteins (HDL), favoring atherogenesis and increasing cardiovascular risks.

According to the World Health Organization, ${ }^{3}$ cardiovascular disease (CVD) is the world's leading cause of death, making up 30\% of global deaths, almost the same rate found in Brazil. ${ }^{1}$ More than $80 \%$ of all cardiovascular diseases (CVD) are found in medium and low-income countries. ${ }^{4}$ Since women live 5-8 years more than men on average, the absolute number of deaths related to cardiovascular diseases is significantly higher among women. Besides, approximately one in every two women will die in consequence of cardiovascular diseases, surpassing the deaths due to all neoplasias. ${ }^{5}$ Nowadays it is clear that different dietary habits define different aspects of the atherosclerotic process and cardiovascular risk factors -including during the climacteric, among them the plasma lipid levels, resistance to insulin and glucidic metabolism, blood pressure, oxidative damage, endothelial function, and vascular inflammation. Consequently, dietary patterns interfere in the chances of atherosclerotic events. ${ }^{6}$ Due to the above-mentioned situations, this study aims at researching factors associated with the prevalence of dyslipidemia among climacteric women treated in a clinic in southern Brazil.

\section{Method}

The study was performed using the database provided in the research work entitled "Climacteric state and metabolic syndrome with climacteric women treated in a clinic in southern Brazil", which was approved by the Caxias do Sul University Ethics Committee under document no. 124/08. It is a cross-sectional study, whose data collection was performed at the Caxias do Sul University's Multidisciplinary Clinic and Climacteric Care, a reference of the Brazilian Public Health Care System in the municipality of Caxias 
do Sul, in the state of Rio Grande do Sul. Data collection was performed between January 2010 and April 2011. ${ }^{7}$ The sample size was calculated to associate climacteric state and metabolic syndrome. A sample of 687 women aged 40-65 years would allow a 1.6 odds ratio, with a $95 \%$ confidence level, an $80 \%$ statistical power, keeping a non-exposed: exposed ratio of 2:1, where non-exposed were pre-climacteric women, and exposed ones were those in the postclimacteric period. ${ }^{7}$

All interviews were performed at the previously mentioned clinic before the medical appointment and were conducted by trained interviewers. A precoded and pretested standardized survey, with specific socioeconomic, demographic and preceding behavioral and reproductive features were applied. The demographic features were age (in years), skin color (white and non-white) and marital status (married/common-law marriage and not married). Socioeconomic features were education (in full years) and family income (in number of minimum salaries - according to values at the time of research). In the analysis of behavioral features, smoking habits were classified as a smoker, ex-smoker, and non-smoker. As for physical activities, women were classified as sedentary (no regular physical activity) and non-sedentary (at least one physical activity once a week for a minimum of 30 minutes). Reproductive variables included the age of first menstruation (menarche) and the number of previous pregnancies. Laboratory tests needed for defining lipid profiles were the analysis of HDL cholesterol, total cholesterol, and triglycerides. They were collected from the participant patients' medical protocol, provided a time limit of four months. For those who had previous tests, new tests were required by the physician in charge of the clinic, with the patient's consent. The outcome "dyslipidemia" was defined based on the presence of at least one blood test alteration (HDL cholesterol, total cholesterol, and triglycerides). To define climacteric state, the participants were questioned if they still had uterus (cases of hysterectomy were excluded) and the last 12 cycles/months were analyzed. If menstrual cycles were regular, patients were classified as premenopausal. If menstrual flow and periods were irregular, they were classified as perimenopausal. Women who reported having no menstruation (amenorrhea) for at least 12 consecutive months were classified as postmenopausal. $^{2}$

To ensure information quality control, $10 \%$ of the interviews were repeated by phone, with a simplified questionnaire. Some defining questions of this questionnaire, i.e., whose answer had no possibility of change during the time the research was carried out, were made by the research coordinators. Information encoding was also made by research coordination. Data entry followed the double-entry procedure, performed with program EPI-DATA 3.1. Entries were compared and their consistency was analyzed. Bivariate analysis was performed with program STATA 9.0 (Stata Corp., College Station, USA).

The chi-square test was used to assess the existence of an association between two quantitative variables. The bivariate analysis included the outcome with the other features of the sample. The effect of the sample's features over dyslipidemia was calculated through prevalence ratios and their $95 \%$ confidence intervals. Since this outcome is quite frequent among this population, Poisson Regression with robust variance was used for the multivariate analysis. The model analyzed variables that presented $\mathrm{p}$-value $<0.20$ in the bivariate analysis. Demographic and socioeconomic variables were introduced initially, later the reproductive and finally the behavioral ones. Only variables with significance up to $20 \%$ were kept in the multivariate model. In the final analysis, a level of significance of up to $5 \%$ was accepted.

\section{Results}

During the collection period (January 2010 to April 2011), 658 women at the Caxias do Sul University's Multidisciplinary Clinic and Climacteric Care were eligible to join the study, where $41(6.2 \%)$ of them were rejected and $67(10 \%)$ did not complete the laboratory tests. The following results counted 550 women. The age range with the highest prevalence of dyslipidemia was between 56 and 65 years, totaling $92.5 \%(\mathrm{p}=0.04)$. An inverse association between education and dyslipidemia was found. Women with more years of education had a smaller percentage of dyslipidemia compared to the ones with lesser years of education, but it had no statistical significance (Table 1). When reproductive variables were analyzed, a statistically significant increase $(\mathrm{p}=0.01)$ in the prevalence of dyslipidemia in women with three or more pregnancies $(86.3 \%)$ compared to women that were never pregnant or with a single pregnancy $(73.6 \%)$ was noticed. A high prevalence of dyslipidemia was observed in women with premature menarche, corresponding to $87.8 \%$ (11 years old or less). As for the menopausal state, it was observed that women in the postmenopausal period have a higher prevalence of dyslipidemia (88.1\%) compared to premenopausal and perimenopausal periods, where this percentage was lower, $83.3 \%$ and $81.1 \%$ respectively. However, these results were not statistically significant. Women classified as obese according to the body mass index (BMI) presented a higher percentage of dyslipidemia. Among the obese, $86.7 \%$ were dyslipidemic, while among the eutrophic population the percentage was reduced to $75.5 \%(\mathrm{p}=0.02)$. There was a high prevalence of women with abdominal obesity (66.4\%). 86.1\% among these were dyslipidemic, while $79.3 \%$ of women without abdominal obesity were dyslipidemic $(\mathrm{p}=0.04)$.

Although it was not statistically significant, it was observed that women with arterial hypertension were more prone to dyslipidemia that women with normal blood pressure. In Table 2, variables like age, number of pregnancies and nutritional status were associated with the prevalence of dyslipidemia in the multivariate model after the adjustment for marital status, education, menopausal state, and abdominal obesity. Multivariate analysis has found that older, obese women, with a higher number of pregnancies, had a higher prevalence of dyslipidemia, which is $11 \%$ higher among women aged 56-65 years when compared to women aged $40-45$ years $\left(\mathrm{IC}_{95 \%} 1.01\right.$ to 1.21$)$. An increase of 1.16 times on the prevalence of dyslipidemia was found among women with three or more pregnancies concerning to those without pregnancy or with one. As for the nutritional status, the prevalence of dyslipidemia was found to be higher among obese women than among eutrophic ones (RP $1.12 ; \mathrm{IC}_{95 \%} 1.00$ to 1.26 ).

\section{Discussion}

The harmful changes in lipids, which are risk factors for CVD, are sharper after the menopause, with possible increase in the triglyceride levels $(\mathrm{TG} \geq 150 \mathrm{mg} / \mathrm{dL})$, elevations in LDL-C levels $(>100 \mathrm{mg} /$ $\mathrm{dL})$, and reduction in HDL-C $(<40 \mathrm{mg} / \mathrm{dL})$ levels - well-known atherogenic conditions. ${ }^{8}$ An elevation of $1 \%$ in LDL-C levels increases CVD risk by $2 \%$, and a decrease of $1 \%$ in HDL-C levels increases this risk between $2 \%$ and $4.7 \%{ }^{10}$. Six months after menopause, total cholesterol (CT), LDL-C, and TG levels increase between $5 \%$ and $10 \%$, and two years later HDL-C decreases approximately $6 \%{ }^{6}$ 
Table I Sample description and prevalence of dyslipidemia in relation to sociodemographic, reproductive and behavioral variables among climacteric women in a clinic in the city of Caxias do Sul (RS), Brazil $(n=550)$

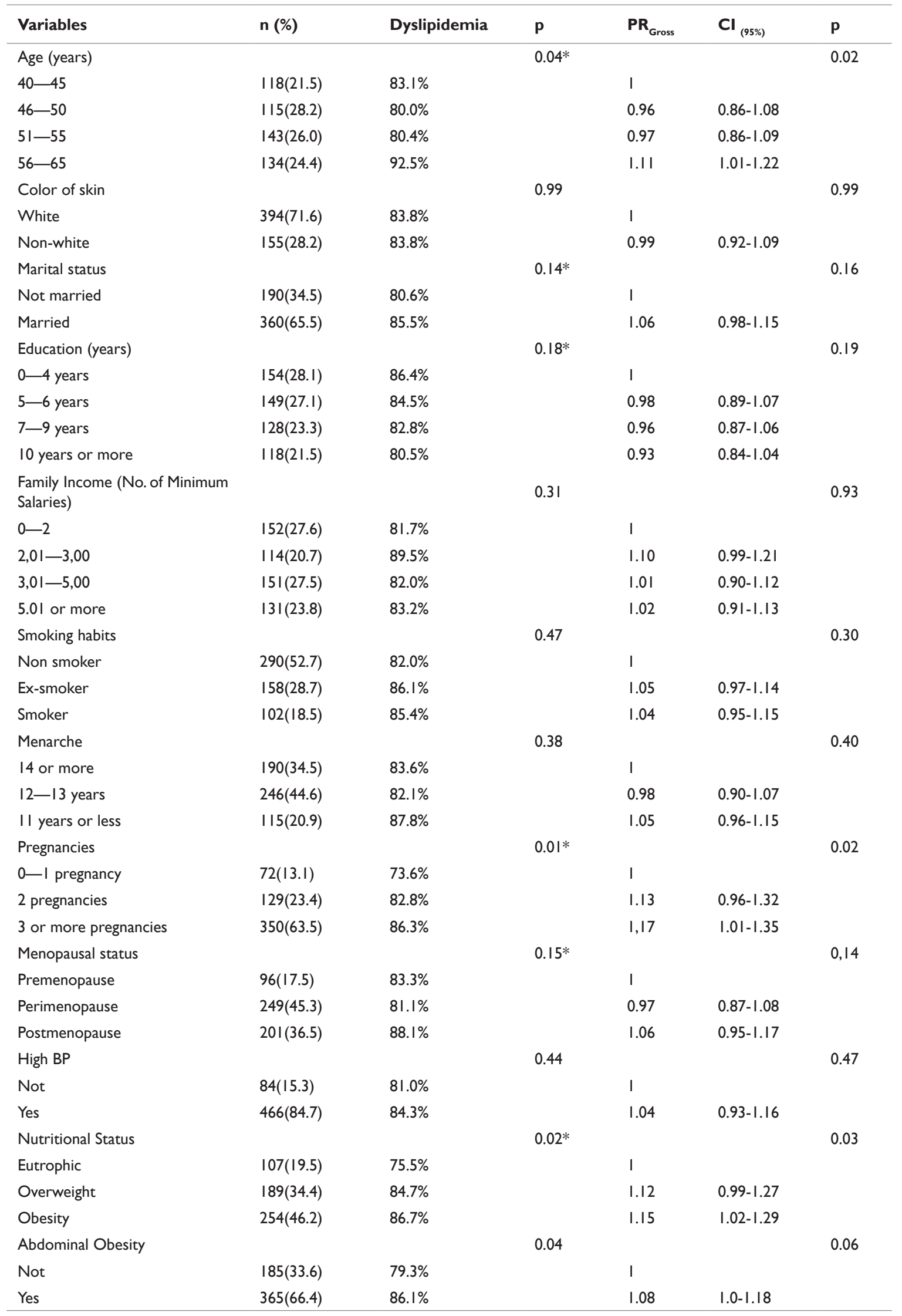


Table 2 Reasons for prevalence of dyslipidemia adjusted in relation to sociodemographic, reproductive and behavioral variables among climacteric women in a clinic in the city of Caxias do Sul (RS), Brazil. $(n=550)$

\begin{tabular}{|c|c|c|c|}
\hline Variables & $\begin{array}{l}\text { PR } \\
\text { Adjusted }\end{array}$ & $\mathrm{Cl}(95 \%)$ & $\mathbf{p}$ \\
\hline Age (years) & & & 0.03 \\
\hline $40-45$ & 1 & & \\
\hline $46-50$ & 0.96 & $0.86-1.07$ & \\
\hline $51-55$ & 0.96 & $0.96-1.07$ & \\
\hline $56-65$ & 1.11 & $|.01-| .21$ & \\
\hline Pregnancy & & & 0.04 \\
\hline 0 - I pregnancy & 1 & & \\
\hline 2 pregnancies & 1.12 & $0.96-\mid .31$ & \\
\hline 3 or more pregnancies & 1.16 & $1.00-1.31$ & \\
\hline Nutritional Status & & & 0.06 \\
\hline Eutrophic & I & & \\
\hline Overweight & I.II & $0.98-1.25$ & \\
\hline Obesity & 1.12 & $1.00-1.26$ & \\
\hline
\end{tabular}

There is a tendency for high dyslipidemia rates among individuals with lower levels of education. Women with up to 4 years of education presented a high percentage of dyslipidemia. According to Wamala et al., ${ }^{9}$ women with a lower educational level had an increased risk for factors associated with cardiovascular diseases and type 2 diabetes. The same result was observed by Oliveira et al., ${ }^{8}$ where the majority of dyslipidemic women (51.1\% of the sample) had fewer years of education. It may be suggested that the lack of education is associated with a higher prevalence of dyslipidemia due to a lesser knowledge of appropriate food intake and healthy habits. This study found that women with three or more pregnancies presented a higher prevalence of dyslipidemia. This result is consistent with a cohort study performed in China with 7,352 women aged 50-93 years. BMI, waist/hip ratio, triglyceride and glucose levels were positively associated with the number of children. ${ }^{10}$ Pregnancy is seen as a factor associated with weight increase for women, possibly due to excessive weight gain during pregnancy, which is related to an increased cardiovascular risk. It is estimated that postpartum weight retention is between $0.5 \mathrm{~kg}$ and $3.8 \mathrm{~kg}$ at each pregnancy. ${ }^{11}$

It was observed that women whose menarche age is equal or inferior to 11 years presented a tendency to higher dyslipidemia levels. Mendonza et al., ${ }^{12}$ studied 1,980 women during postmenopause and found an increased risk for higher hypercholesterolemia levels, overweight, and obesity among those whose menarche happened when they were 11 years old or less. Such results are consistent with the study performed with 9,097 Chinese women, which also observed that early menarche was associated with an abdominal fat increase, higher triglyceride and total cholesterol levels, as well as with a decrease of HDL cholesterol in adult women. ${ }^{13}$ This study observed that $88.1 \%$ of the women classified in postmenopause presented dyslipidemia, although it was not statistically significant. Piazza, De Lorenzi e Saciloto. ${ }^{14}$ also observed a higher percentage of dyslipidemia among postmenopausal women, and the average of their total cholesterol levels was $237 \mathrm{mg} / \mathrm{dL}$, whereas among premenopausal women the average came down to $216 \mathrm{mg} / \mathrm{dL}$. Besides the tendency to gain weight, postmenopausal women are also prone to changes in their lipid metabolism, due to estrogenic deprivation, which elevates the total cholesterol, lipoproteins and triglycerides levels, leading to a lipid profile that is highly favorable to atherogenesis in this population, particularly when associated with diabetes mellitus and hypertension Pasquali et al. ${ }^{15}$ Literature has outlined climacteric, particularly the postmenopause period, as the most risky period in terms of cardiovascular diseases. Therefore, it is extremely important to know the age in which menopause comes to different populations. ${ }^{16}$

Regarding nutritional status, in the present study, obese women presented a higher prevalence of dyslipidemia. According to Oliveira et al., ${ }^{8}$ it was observed that BMI and the waist circumference were significantly associated with dyslipidemia during climacteric. Among women with dyslipidemia, there was a significantly more elevated proportion of weight excess ( $42.2 \%$ overweight and $31.1 \%$ obesity) and abdominal obesity than among women with no dyslipidemia. In another study with women aged 45-54 years, which included women in premenopause, in the menopause transition and postmenopause periods, it was observed that changes in the concentration of triglycerides and LDL cholesterol are associated with age and to BMI, while changes in HDL cholesterol are associated only with BMI. ${ }^{17}$

In the present study, it was observed that $66.4 \%$ of women presented abdominal obesity and among these women $86.1 \%$ were dyslipidemic. In a study performed in Scotland with postmenopausal women who did not make use of hormone replacement, a positive correlation between waist circumference and serum concentrations of insulin \& triglycerides was verified, as well as negative correlations among this anthropometric variable and HDL cholesterol, even among eutrophic women, according to BMI. ${ }^{18}$ Another study, performed in a climacteric clinic with 518 women aged $45-65$ years, verified a significant association between waist circumferences above $80 \mathrm{~cm}$ and dyslipidemia. ${ }^{19-22}$

Finally, it was observed that climacteric is strongly linked to several hormonal changes, including the increase of dyslipidemia prevalence, which can be strengthened with increased age, obesity, and increased number of pregnancies. We emphasize the importance of conducting longitudinal studies with women from the beginning of reproductive life for further clarification on the relationship between hormonal aspects and dyslipidemia. Studies that consider women's sociodemographic and lifestyle characteristics may provide insights for a better understanding of this relationship and contribute to the proposition of preventive measures as a guide public health policies.

\section{Acknowledgments}

None.

\section{Conflicts of interest}

All authors declare no conflict of interest.

\section{Funding details}

None.

\section{References}

1. Nosse TM, Moreira SLN, Andrade KC. Dietary evaluation of climacteric women treated at a nutrition clinic-school in São Paulo. Rev Bras Ciências da Saúde. 2009;7(21):26-31.

2. Gallon CW. Nutritional profile and quality of life of climacteric women. Rev Bras Ginecol Obstet. 2012;34(4):175-183. 
3. World Health Organization (WHO). Cardiovacular Diseases (CVDs). Fact Sheet $n^{\circ} 317 ; 2011$.

4. Brasil. Ministry of Health. DATASUS. Indicators and basic data. Brasil; 2009.

5. Medeiros SF. Risk and assistance to dyslipidemia in climacteric. Revista Brasileira de Ginecologia e Obstetrícia. 2008;30(12):591-593.

6. Santos RD, Gagliardi AC, Xavier HT, et al. Guideline on Fat Consumption and Cardiovascular Health. Brazilian Society of Cardiology. Arq Bras Cardiool. 2013;100;(Supl 3):1-40.

7. Theodoro $\mathrm{H}$, Rodrigues $\mathrm{AD}$, Mendes $\mathrm{KG}$, et al. Reproductive characteristics and obesity in middle-aged women seen at an outpatient clinic in southern Brazil. Menopause. 2012;19(9):1022-1028.

8. Oliveira TR, Sampaio HA, Carvalho FH, et al. Factors associated with dyslipidemia in postmenopausal. Rev Bras Ginecol Obstet. 2008;30(12):594-601.

9. Wamala SP, Lynch J, Horsten M, et al. Education and the metabolic syndrome in women. Diabetes Care. 1999;22(12):1999-2003.

10. Lao XQ, Thomas GN, Jiang CQ, et al. Parity and the metabolic syndrome in older Chinese women: The Guangzhou Biobank cohort Study. Clinical Endocrinology. 2006;65(4):460-469.

11. Blaudeau TE, Hunter GR, Sirikul B. Intra-abdominal adipose tissue deposition and parity. Int J Obes (Lond). 2006;30(7):1119-1124.

12. Mendoza N, Galliano D, Salamanca A, et al. Lowering the age at menarche and risk of early menarche in a population of Spanish postmenopausal women during the past two decades. Menopause Int. 2010;16:111-114.

13. Feng Y, Hong X, Wilker E, et al. Effects of age at menarche, reproductive years, and menopause on metabolic risk factors for cardiovascular diseases. Atherosclerosis. 2008;196(2):590-597.
14. Piazza IP, De Lorenzi DR, Saciloto B. Cardiovascular risk assessment among climacteric women attending a family health program. Rev Gaúcha Enferm. 2005;26(2):200-209.

15. Pasquali R, Casimirri F, Pascal G, et al. Influence of menopause on blood cholesterol levels in women: the role of body composition, fat distribution and hormonal milieu. Virgilio Menopause Health Group. $J$ Intern Med. 1997;241(3):195-203.

16. Sociedade Brasileira de Diabetes. Diagnosis and classification of diabetes mellitus and treatment of type 2 diabetes mellitus: recommendations of the Brazilian Society of Diabetes. Arquivos Brasileiros de Endocrinologia e Metabologia. 2000;44(Suppl 1):S8-S35.

17. O'brien T, Nguyen TT. Lipids and lipoproteins in women. Mayo Clin Proc. 1997;72:235.

18. Van Pelt RE, Evans EM, Schechtman KB, et al. Waist circumference vs body mass index for prediction of disease risk in postmenopausal women. Int J Obes Relat Metab Disord. 2001;25(8):1183-1188.

19. Raskin DBF, Pinto-Neto AM, Paiva LHSC, et al. Anthropometric profile, menopausal status and metabolic changes associated with cardiovascular disease. Rev Ciênc Méd. 2003;12(3):215-226.

20. Mendes KG, Theodoro H, Rodrigues AD, et al. Prevalence of metabolic syndrome and its components in menopausal transition: a systematic review. Cadernos de Saúde Pública (ENSP Impresso). 2012;28:14231437.

21. The Sixth Report of the Joint National Committee on Prevention, Detection, Evaluation and Treatment of High Blood Pressure. Arch Intern Med. 1997;157(21):2414-2446.

22. Torng PL, Su TC, Sung FC, et al. Effects of menopause on intraindividual changes in serum lipids, blood pressure, and body weigth - the ChinShan Community Cardiovascular Cohort Study. Atherosclerosis. 2002;61(2):409-415. 